\title{
Wind Shear for Small Thickness Layers ${ }^{1}$
}

\author{
Manuel Armendariz and Laurence J. Rider \\ Atmospheric Sciences Laboratory, White Sands Missile Range, N. Mex. \\ (Manuscript received 19 April 1966, in revised form 5 July 1966)
}

\begin{abstract}
Wind shear in the lowest $10,000 \mathrm{ft}$ of the atmosphere was investigated utilizing two or more cinetheodolites (Contraves cameras) tracking a smooth spherical 100 -gm balloon. These data indicate that a simple power law relationship exists between 1) layer thickness and the magnitude of the vector shear, 2) layer thickness and the magnitude of the mean extreme vector shear, and 3) magnitude of the mean shear and standard deviation for given layers. From this relationship it appears that wind shears for small layers $(50,100$, $200 \mathrm{ft}$ ) may be derived from wind values obtained over large shear intervals $(1000,2000,3000 \mathrm{ft})$ with present operational equipment such as the GMD-1.

A relationship exists between wind speed and wind shear for various shear intervals such that as the wind speed increases to $30-40 \mathrm{ft} \mathrm{sec}^{-1}$, the mean shear increases; the shear decreases for higher wind speeds.
\end{abstract}

\section{Introduction}

Wind shear is generally computed from wind data obtained utilizing instruments such as GMD-1, radar, theodolites, etc. Due to the inaccuracies of the wind data as obtained with these instruments, meaningful wind shear calculations are limited to a layer thickness of over $1000 \mathrm{ft}$. Leviton (1962) stated that with special balloons and the FPS-16 radar, precise measurements of wind can be made for 100 -ft layers. However, Scoggins (1962) reports a maximum shear error of approximately $3.9 \mathrm{ft} \mathrm{sec}^{-1}$ for a 164-ft layer $(0.084$ $\left.\sec ^{-1}\right)$. This error is of the order of the wind shear which is being measured. Moreover, the expense involved in utilizing an FPS-16 radar on a routine basis for the purpose of tracking a balloon is prohibitive.

The purpose of this report is to determine if a power law relationship, as advanced by Essenwanger (1963) at Cape Kennedy, exists at White Sands Missile Range between layer thickness and shear. Wind data used to investigate this relationship were obtained with Contraves cameras tracking a smooth spherical 100-gm balloon and the results shown will apply only to shears computed from similar rising balloons.

\section{Relationship between layer thickness and mean shear}

Essenwanger (1963) suggested that, since the wind profile does not change linearly with height and random fluctuations distort the mean wind profile, an equation of the type

$$
\frac{\bar{\gamma}_{1}}{\bar{\gamma}_{2}}=C_{0} \frac{\Delta h_{1}}{\Delta h_{2}}
$$

1 Presented at the AMS/AIAA Conference on Aerospace Meteorology, Los Angeles, Calif., 28-31 March 1966. where $\gamma$ is the magnitude of the difference in wind velocity (shear) between two levels (not to be confused with magnitude of vectorial wind shear $\mathbf{W}_{\mathrm{s}}$, which is $\gamma(\Delta h), \Delta h$ is layer thickness, and $C_{0}$ a parameter which varies with climatic conditions, would best represent the shear. Since this implies that the relationship between layers is independent of the initially chosen size of $\Delta h_{1}$, one would expect that a change in shear interval from 1000 to $500 \mathrm{ft}$ would have the same effect as a change from 100 to $50 \mathrm{ft}$.

To express the above, a modification of Eq. (1) should be written as

$$
\frac{\bar{\gamma}_{1}}{\bar{\gamma}}=C_{0}\left(\frac{\Delta h_{1}}{\Delta h}\right)^{a_{s}}
$$

which can be transformed into the general form

$$
\bar{\gamma}_{\Delta h}=a_{0}(\Delta h)^{a_{1}},
$$

where $a_{1}$ denotes the power by which the decrease or increase within shear intervals is established and $a_{0}$ is a new parameter, variable with climatic conditions.

Essenwanger assumed a linear relationship between $\bar{\gamma}$ and its standard deviation $\sigma$, and empirically showed this relationship to be

or

$$
\begin{gathered}
\sigma=A_{0}+A_{1} \bar{\gamma}, \\
\sigma=C(\Delta h)^{a_{1}}+A_{0},
\end{gathered}
$$

where $C=a_{0} a_{1}$ with $a_{0}, a_{1}, A_{0}$ and $A_{1}$ being variable with climatic conditions.

\section{Data collection and reduction}

During August and September 1964, 68 balloons $(100 \mathrm{gm})$ were tracked by two or more Contraves 
cameras to altitudes of approximately $10,000 \mathrm{ft}$ above the surface. Framing rate of the balloon by each camera was one per second (approximately one every $17 \mathrm{ft}$ in height). The shutters of the cameras opened simultaneously by electrical pulses from a central timing station. Each frame contained the elevation and azimuth angle, time and balloon position referenced to a cross index. This synchronous photo-recording of the target and the cross index allows subsequent recognition and consideration of tracking errors.

The mathematical solution of balloon position was derived by Babcock (1964). Briefly this solution consists of the following:

1) The azimuth and elevation angle data are corrected for eccentricity, tracking, level and tilt, orientation, etc.

2) A 2-station Bodwell solution which minimizes the squares of the distance from the line of sight to a point is used as a first approximate point. This point and other points computed from a 2-station solution are then used in the Davis solution which minimizes the squares of the azimuth and elevation angle residuals for each station.

3) Balloon position is thus derived for each second and wind velocity computed by least-squares solution over a 7 -sec interval.

4) Wind velocity data are then utilized to compute wind shear for $200-\mathrm{ft}$ thickness layers up to $3000-\mathrm{ft}$ layers, i.e., $200,400,600, \ldots, 3000 \mathrm{ft}$. Thus, if the balloon rises at $17 \mathrm{ft} \mathrm{sec}$, the 200 -ft layer shears are for approximate 12-sec intervals whereas the $400-\mathrm{ft}$ layers are for a 24 -sec interval, etc. Shears are computed by differentiating the velocities at the top and bottom of the layer.

5) The wind shear values are then used to compute means, standard deviation and maximum shears.

6) These data are then applied to a simple power law (Eq. 3) in order to solve for $a_{0}$ and $a_{1}$.

\section{Discussion}

Since wind shear is computed from wind data, the errors inherent in the system with which the wind data are collected must be minimized so that the computed shears are valid. Photographic recording of the elevation and azimuth angles along with the balloon position relative to a frame index ensures a very accurate measurement of angles since the recorded angles can be corrected for the inherent and tracking errors.

The problem of aerodynamically induced horizontal motions of a smooth spherical balloon has been extensively investigated by Scoggins (1964, 1965), Murrow and Henry (1965), MacCready and Jex (1964), MacCready (1965), and others. These self-induced balloon oscillations may produce significant errors in wind velocities. According to Scoggins, this error is a function of averaging time. Thus, the error in wind shear calculation may be expected to increase as the thickness of the layer is decreased. These errors are considered significant for layer thicknesses less than $200 \mathrm{ft}$. Thus, it becomes advisable to compute the shear for larger layers and to apply a power law equation to these values to compute shears for smaller layers.

To generate the plotted points for Fig. 1, shear $\bar{\gamma}$, calculated for different layer thickness, i.e., 200, 400, $600 \mathrm{ft}$, etc., was summed throughout the entire 10,000 $\mathrm{ft}$ and averaged for each layer. For example, the summation of the $200-\mathrm{ft}$ layers resulted in 50 values of shear, multiplied by the number of observations (68) or approximately 3400 samples, to determine the mean shear $\bar{\gamma}$ and the standard deviation $\sigma$ for the $200-\mathrm{ft}$ layer. Similar treatment was given the other layers using end points for the differentiation.

It will be noted that the mean shear for White Sands Missile Range (WSMR) is generally twice the value reported at Cape Kennedy by Essenwanger and Billions (1965). There are several possible reasons for this, among them being:

1) The wind data used by Essenwanger to generate curve 1 were obtained from rockets by determining the angle-of-attack. Reisig (1956) states that this wind measurement is instantaneous and continuous. Rachele and Veith (1965) state that "A continuously measured instantaneous profile along the trajectory (of a rocket), while considered the ultimate and highly desirable, is impractical at the present state-of-the-art . .." This statement is based on the fact that if rockets responded instantly to all frequencies of wind it would be impossible to make a reasonable impact prediction for an unguided rocket. Since satisfactory impacts

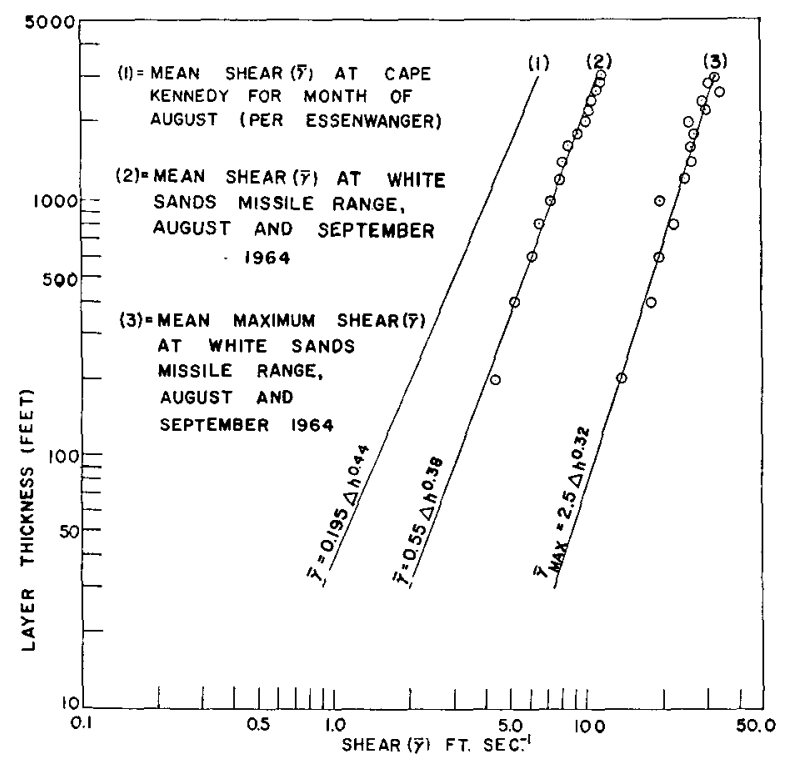

FIG. 1. Relationship between layer thickness $\Delta h$ and shear $\bar{\gamma}$. 
have been obtained in the firing of unguided rockets at WSMR, it is reasonable to assume that a rocket does not respond to all frequencies of wind and that the wind data oblained by Reisig are not instantaneous values but, in fact, are smoothed values. This smoothing would tend to filter out the high frequencies and give a lower value of wind shear.

2) The wind data used for curve 2 were obtained utilizing 100 -gm smooth spherical balloons. According to Scoggins (1965), at altitudes below $5000 \mathrm{ft}$, these balloons operate at a supercritical Reynolds number. This means that there are horizontal self-induced oscillations of the balloon which change the amplitude of the wind perturbations in the real atmosphere. This would tend to increase the mean shear value for WSMR.

3) Finally, the fact that the wind field over mountainous terrain is generally affected to great heights would suggest that the mean wind shear at WSMR should be greater than that found over or near a water surface, such as at Cape Kennedy.

It is felt that the last factor mentioned above is the largest contributor to the difference in the two curves. This is somewhat substantiated by the steeper slope of the curve a.t WSMR. Despite the differences in the two curves, the functional relationship between the layer thickness and the shear is not altered.

The slope of the mean maximum shear, curve 3 , is slightly steeper than the mean shear curve as found by Essenwangrer and Billions (1965). The dispersion of some of the points along this line may be attributed to the self-induced horizontal motion of the balloon which tends to magnify the extreme values of shear.

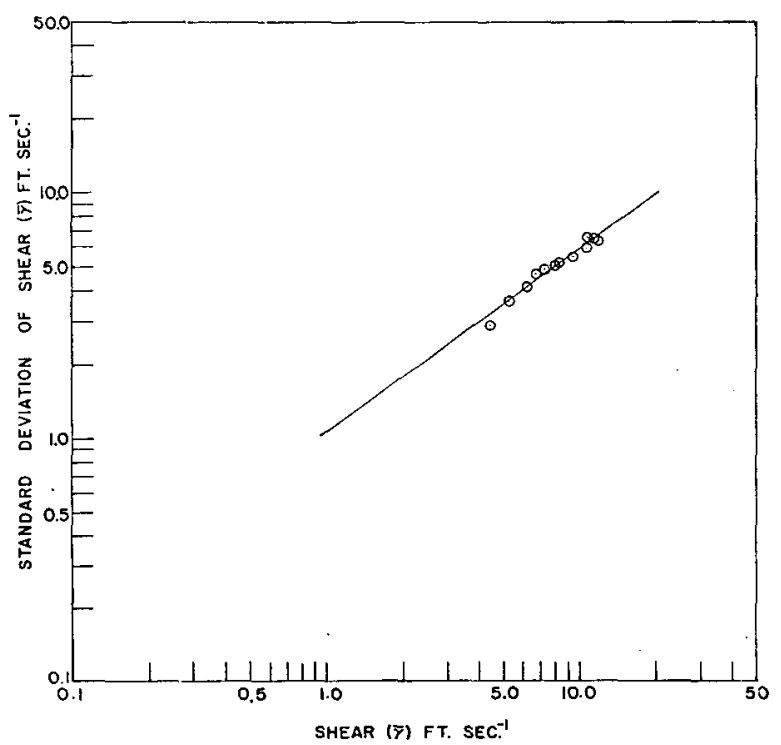

FIG. 2. R.elationship between the standard deviation shear and the shear.

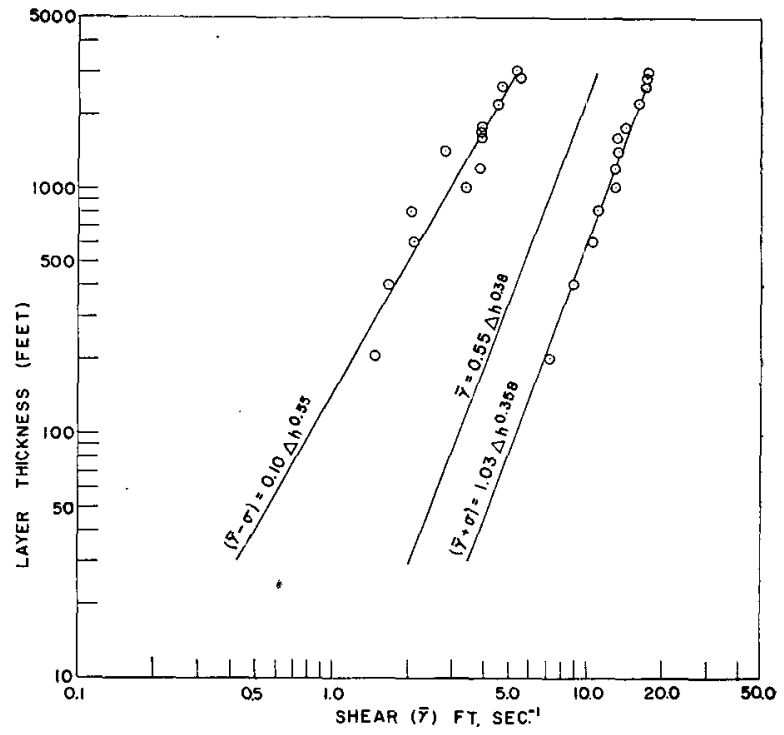

FIG. 3. Dispersion of shear $\bar{\gamma}$ for given layer thicknesses.

Fig. 2, which shows an exponential relationship of the dispersion of shear to the value of shear, does not bear out the linear relationship of Eq. (4) as assumed by Essenwanger. The same factors which are given for the difference in mean shear curves at Cape Kennedy and at WSMR are considered the cause of this difference in relationship. However, it should be expected that as shear increases the standard deviation of shear should also increase, but only to a limited degree, since the atmosphere is capable of sustaining only a certain amount of turbulence. The equation of the curve,

$$
\sigma_{\gamma}=1.1 \bar{\gamma}^{0.74}
$$

can be written in terms of layer thickness by substituting (3) into (6) such that

$$
\sigma_{\gamma}=k_{0} \Delta h^{k_{1}}
$$

where $k_{0}=1.1 a_{0}^{0.74}$ and $k_{1}=0.74 a_{1}$. Thus, the relationship of standard deviation of shear for a given layer thickness as a function of the layer thickness is established.

Fig. 3, which depicts the dispersion of shear about the mean, can be used as a limiting graph for the prediction of shear. For example, on 24 August 1964, the mean shear $\bar{\gamma}$ for $1000-\mathrm{ft}$ layers was $3.1 \mathrm{ft} \mathrm{sec}-1$. This value falls within the one sigma value for the $1000-\mathrm{ft}$ layer. Using $3.1 \mathrm{ft} \mathrm{sec}^{-1}$ and Eq. (3) with $a_{1}=0.38$, we find a value of $1.68 \mathrm{ft} \mathrm{sec}^{-1}$ for the $200-\mathrm{ft}$ layer thickness. The actual observed value was 1.74 or a difference of $0.06 \mathrm{ft} \mathrm{sec}-1$. Essenwanger, in predicting shears for 314-ft layers using 1570-ft layers, found differences of $0.13-1.1 \mathrm{ft} \mathrm{sec}^{-1}$ between the value computed by Eq. (3) and the observed value, for $a_{1}=0.5$. It should 


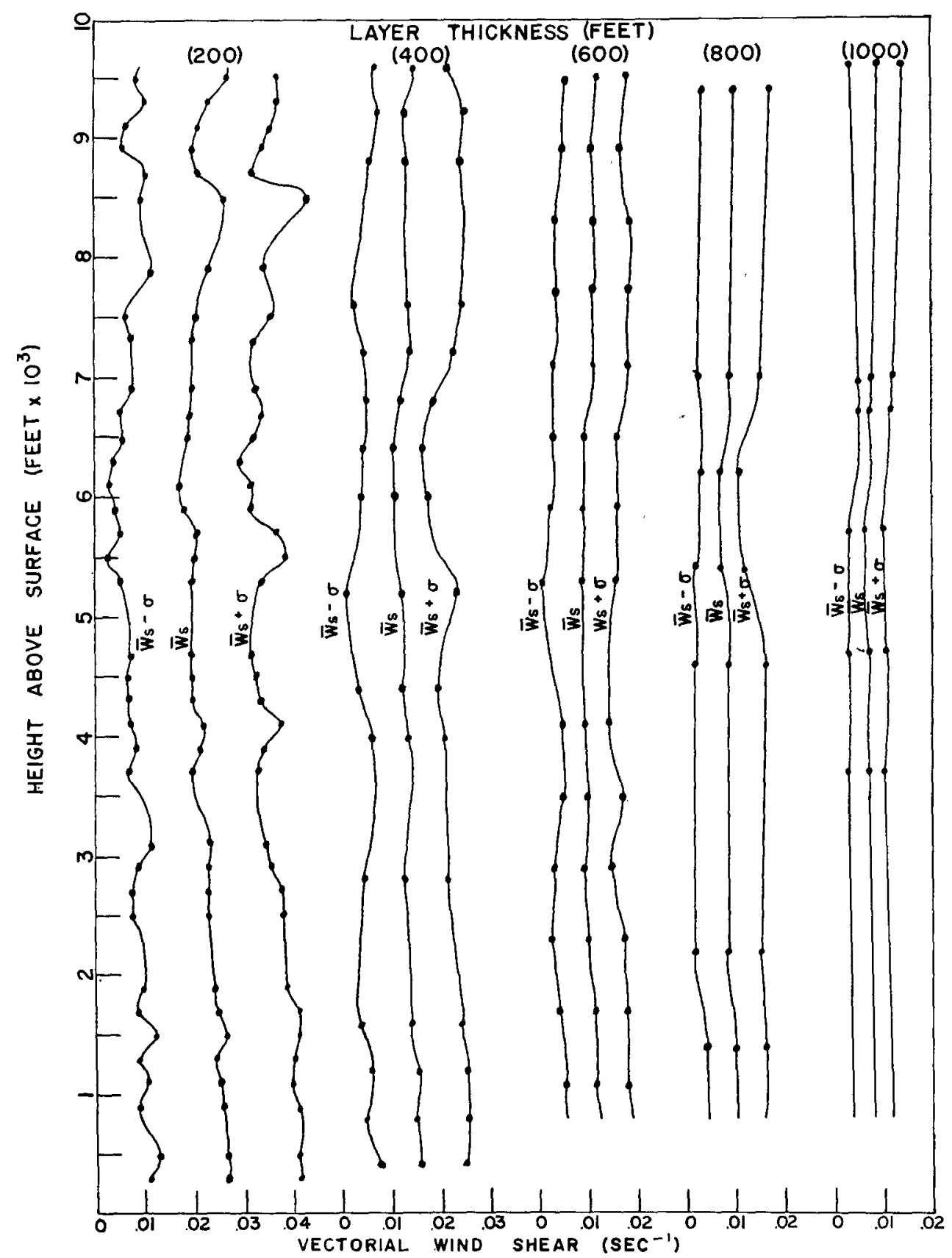

Fig. 4. Dispersion of the magnitude of vectorial wind shear $\mathbf{W}_{\mathbf{s}}$ for various layer thicknesses. $\left|\mathbf{W}_{s}\right|=\bar{\gamma} / \Delta h$.

be noted from Fig. 5 that these values are within one sigma of the mean shear.

The reason for keeping $a_{1}$ constant and computing $a_{0}$ from (3) is that the slope of the curves does not significantly change from day to day, whereas $a_{0}$ appears to be a function of the stability of the atmosphere. For example, the exponent $a_{1}$ for the mean shear at Cape Kennedy was 0.44 whereas at White Sands it was
0.38 . This value appears to vary from 0.3 for extreme shears to 0.5 for the mean shear. $A_{0}$, however, appears to vary from approximately 0.08 for the mean shear to 2.5 for the maximum shear. Essenwanger found that a prediction scheme in which the slope of the mean shear for a given month is used to predict the shear for small layers yields better results. He also concluded that the best prediction result is obtained by employing the 
distribution of shear for layer thickness closest to the desired prediction interval. However, it should be stated that selection of the shear layer is limited to the instrumentation used to obtain the data from which shear is calculated.

Fig. 4 dep.cts the magnitude of vectorial wind shear vs. height for various layer thicknesses. As would be expected, the dispersion of wind shear for the $200-\mathrm{ft}$ layer is about three times that of the 1000 -ft layers. It is interesting to note that the mean vectorial wind shear does not change significantly with height for any of the layer thickresses presented. Also, it appears that stationarity is reached when computing the $1000-\mathrm{ft}$ layer wind shear, which would suggest that any prediction scheme dependent upon the calculation of wind shear for srnall layer thicknesses should utilize the 1000 -ft layer. This may be only a characteristic of the instrumentation used in this study and should be investigated for other instruments, such as the GMD.

The results plotted in Fig. 4 compare favorably with those obtained by Dvoskin and Sissenwine (1958). For example, they found that for 91 per cent of the time, wind shear for $1000-\mathrm{ft}$ increments was between 0.000 to 0.019 , whereas a two-sigma value for the data presented here ranges from 0.000 to 0.017 .

Fig. 5 is a graph of vectorial wind shear as a function of speed for the first $10,000 \mathrm{ft}$ of the atmosphere. The slight drop in mean vectorial shear from the $0-10$ to the 11-20 feet $\sec ^{-1}$ wind may be caused by the extreme

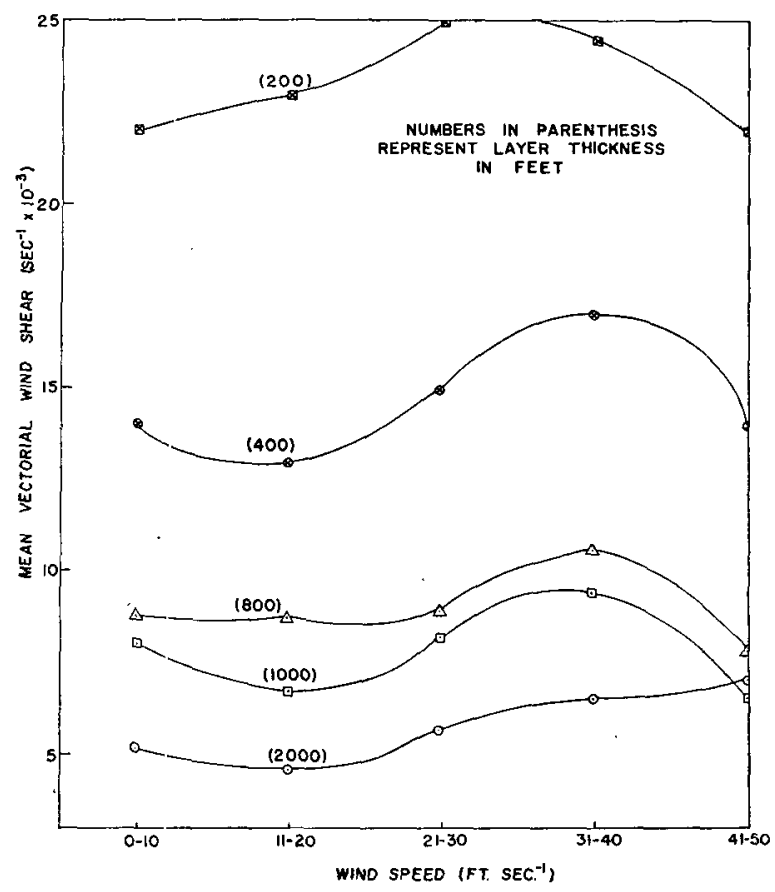

FIG. 5. Vectorial wind shear as related to wind speed for various layer thicknesses. wind direction changes which occur at the lower wind speeds. However, there is a rise in wind shear as the wind speed increases to approximately $31-40 \mathrm{ft} \mathrm{sec}^{-1}$. The decrease in wind shear with the $41-50 \mathrm{ft} \mathrm{sec}^{-1}$ wind suggests that as the wind increases there is a tendency for the complete profile (up to $10,000 \mathrm{ft}$ ) to assume the existing wind speed.

\section{Conclusion}

Calculation of wind shear for small thickness layers $(50,100$ and $200 \mathrm{ft})$, utilizing shear data from layer thicknesses of $1000 \mathrm{ft}$ or greater, has led to the conclusion that a simple power law relationship exists between layer thicknesses and mean wind shear and/or maximum shear. Since the exponent in the power law does not change significantly on a day-to-day basis, it can be used to compute wind shear for the smaller layer once the exponential relationship is established for a given area and climatic conditions. The calculated value of the exponent for the months of August and September at WSMR was 0.32 for the mean maximum shear and 0.38 for the mean shear.

An exponential relationship also exists between wind shear and its standard deviation. The value of the exponent at WSMR for August and September was 0.74 .

The relationship of wind shear to wind speed revealed an increase in shear as wind speed increases to approximately 30-40 ft sec-1 . A decrease in wind shear occurs after the $30-40 \mathrm{ft} \mathrm{sec}^{-1}$ increment, suggesting homogeneity of the atmosphere at least in the first $10,000 \mathrm{ft}$ at the higher wind speeds.

\section{REFERENCES}

Babcock, Virge A., 1964: N-station Askania-Contraves program. Data Reduction Directorate, Computing Division, White Sands Missile Range, N. Mex., Internal Report, 30 pp.

Dvoskin, Norman, and Norman Sissenwine, 1958: Evaluation of AN/GMD-2 wind shear data for development of missile design criteria. Air Force Surveys in Geophysics, No. 99, Air Force Cambridge Research Laboratories, AFCRC-TN-58$259,72 \mathrm{pp}$.

Essenwanger, O., 1963: On the derivation of frequency distributions of vector wind shear values for small shear intervals. Geofis. Pura Appl., 56, 216-224.

smaller shear intervals. Report TP-65-4, U. S. Army Missile Command, Redstone Arsenal, Ala., AD-466 161, 28 pp.

Leviton, Robert, 1962: A detailed wind profile sounding technique. Proc. National Symposium on Winds for Aerospace Vehicle Design, 1, 187-196. Also, Air Force Surveys in Geophysics, No. 140, Air Force Cambridge Research Laboratories, AD-146 858, 10 pp.

MacCready, Paul B., Jr., 1965: Comparison of some balloon techniques. J. Appl. Meteor., 4, 504-508.

—, and H. R. Jex, 1964: Study of sphere motion and balloon wind sensors. NASA TM-X-53089, $48 \mathrm{pp}$.

Murrow, Harold N., and Robert N. Henry, 1965: Self-induced balloon motions. J. Appl. Meteor., 4, 131-138. 
Rachele, Henry, and D. R. Veith, 1965: Surface wind sampling for unguided rocket impact prediction. ERDA-255, U. S. Army Electronics Research and Development Activity, White Sands Missile Range, N. Mex., AD-456 795, 54 pp. Reisig, G., 1956: Instantaneous and continuous wind measurements up to the higher stratosphere. J. Meteor., 13, 448455 .
Scoggins, James R., 1962: An evaluation of detail wind data as measured by the FPS-16 radar/spherical balloon technique. NASA MTP-Aero 62-38.

- 1964 : Aerodynamics of spherical balloon wind sensors. J. Geophys. Res., 69, 591-598.

- 1965 : Spherical balloon wind sensor behavior. J. Appl. Meteor., 4, 139-145. 